\title{
Dialysis initiation, modality choice, access, and prescription: conclusions from a Kidney Disease: Improving Global Outcomes (KDIGO) Controversies Conference
}

\author{
Christopher T. Chan ${ }^{1}$, Peter J. Blankestijn², Laura M. Dember ${ }^{3}$, Maurizio Gallieni ${ }^{4}$, David C.H. Harris ${ }^{5}$, \\ Charmaine E. Lok ${ }^{7}$, Rajnish Mehrotra ${ }^{6}$, Paul E. Stevens ${ }^{7}$, Angela Yee-Moon Wang ${ }^{8}$, Michael Cheung ${ }^{9}$, \\ David C. Wheeler ${ }^{10}$, Wolfgang C. Winkelmayer ${ }^{11}$ and Carol A. Pollock ${ }^{5}$; for Conference Participants ${ }^{12}$ \\ ${ }^{1}$ University Health Network, University of Toronto, Ontario, Canada; ${ }^{2}$ Department of Nephrology and Hypertension, University Medical \\ Center Utrecht, Utrecht, The Netherlands; ${ }^{3}$ Renal-Electrolyte and Hypertension Division, Perelman School of Medicine, University of \\ Pennsylvania, Philadelphia, Pennsylvania, USA; ${ }^{4}$ Department of Clinical and Biomedical Sciences "Luigi Sacco", University of Milan, Milan, \\ Italy; ${ }^{5}$ University of Sydney, Sydney, NSW, Australia; ${ }^{6}$ Division of Nephrology, Kidney Research Institute and Harborview Medical Center, \\ University of Washington, Seattle, Washington, USA ${ }^{7}$ Kent Kidney Care Centre, East Kent Hospitals, University NHS Foundation Trust, \\ Canterbury, Kent, UK; ${ }^{8}$ Department of Medicine, Queen Mary Hospital, University of Hong Kong, Hong Kong, China; ${ }^{9} \mathrm{KDIGO}$, Brussels, \\ Belgium; ${ }^{10}$ University College London, London, UK; and ${ }^{11}$ Selzman Institute for Kidney Health, Section of Nephrology, Department of \\ Medicine, Baylor College of Medicine, Houston, Texas, USA
}

Globally, the number of patients undergoing maintenance dialysis is increasing, yet throughout the world there is significant variability in the practice of initiating dialysis. Factors such as availability of resources, reasons for starting dialysis, timing of dialysis initiation, patient education and preparedness, dialysis modality and access, as well as varied "country-specific" factors significantly affect patient experiences and outcomes. As the burden of end-stage kidney disease (ESKD) has increased globally, there has also been a growing recognition of the importance of patient involvement in determining the goals of care and decisions regarding treatment. In January 2018, KDIGO (Kidney Disease: Improving Global Outcomes) convened a Controversies Conference focused on dialysis initiation, including modality choice, access, and prescription. Here we present a summary of the conference discussions, including identified knowledge gaps, areas of controversy, and priorities for research. A major novel theme represented during the conference was the need to move away from a "one-size-fits-all" approach to dialysis and provide more individualized care that incorporates patient goals and preferences while still maintaining best practices for quality and safety. Identifying and including patient-centered goals that can be validated as quality indicators in the context of diverse health care systems to

\footnotetext{
Correspondence: Christopher T. Chan, Division of Nephrology, University Health Network, 200 Elizabeth Street, 8N room 846, Toronto, ON M5G 2C4, Canada. E-mail: christopher.chan@uhn.ca; or Carol A. Pollock, The University of Sydney School of Medicine, Kolling Institute of Medical Research, Royal North Shore Hospital, Pacific Hwy, St. Leonards NSW 2065, Australia. E-mail: carol.pollock@sydney.edu.au

${ }^{12}$ See Appendix for list of other conference participants.
}

Received 20 September 2018; revised 21 December 2018; accepted 4 January 2019 achieve equity of outcomes will require alignment of goals and incentives between patients, providers, regulators, and payers that will vary across health care jurisdictions.

Kidney International (2019) घ, घ-目; https://doi.org/10.1016/

j.kint.2019.01.017

KEYWORDS: goal-directed dialysis; hemodialysis; home dialysis; initiation; modality; peritoneal dialysis; prescription; symptom control; vascular and peritoneal dialysis access

Copyright @ 2019 The Authors. Published by Elsevier Inc., on behalf of the International Society of Nephrology. This is an open access article under the CC BY-NC-ND license (http://creativecommons.org/licenses/by-nc-nd/4.0/).

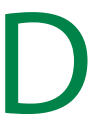
uring the past 3 decades, the number of persons undergoing maintenance dialysis globally has increased dramatically. ${ }^{1}$ In 2010 it was estimated that the number of patients on dialysis was more than 2 million worldwide, and modeling data suggest this number will more than double by $2030 .{ }^{2}$ Several factors have contributed to the increase: improved survival of the general population, reduction in mortality of dialysis patients, an increase in the incidence of chronic kidney disease (CKD), broadening of kidney replacement therapy acceptance criteria, and greater access to maintenance dialysis in low- and middle-income countries. $^{1,3-5}$

The circumstances of dialysis initiation and the choices regarding initial modality and access can significantly affect patient experiences and outcomes. Lack of patient preparedness and an urgent start to dialysis are associated with lower survival and higher morbidity. ${ }^{6,7}$ Home modalities such as home hemodialysis and peritoneal dialysis can improve patients' perception of autonomy. ${ }^{8}$ Lower mortality, fewer medical complications, and lower costs are associated with hemodialysis vascular access via an arteriovenous fistula (AVF) versus 
arteriovenous graft (AVG) or central venous catheter (CVC). ${ }^{4}$ Yet in some circumstances-such as in older patients or those with poor arteriovenous access-an AVG or CVC may be preferred.

Historically, the evaluation of "dialysis adequacy" has been based on small solute clearance. This limited focus excludes the multidimensional parameters involved in achieving optimal dialysis and overlooks necessary evaluations that reflect the many comorbidities present in the dialysis population and how well or how satisfied the patients feel about their treatment. Patients and clinicians can have divergent and sometimes conflicting goals for hemodialysis treatment, with clinicians focused on outcomes such as mortality and biochemical markers and patients prioritizing their well-being and lifestyle. ${ }^{9}$ For example, some patients on home hemodialysis have reported a willingness to trade months of survival for ability to travel. ${ }^{10}$ With the increasing recognition of the importance of patient preferences and satisfaction for shared decision-making and assessing outcomes, ${ }^{8,9,11-17}$ it has become clear that a more multifaceted approach is needed for evaluating dialysis as a treatment modality. ${ }^{18}$

In January 2018, KDIGO (Kidney Disease: Improving Global Outcomes) convened a Controversies Conference titled "Dialysis Initiation, Modality Choice, Access, and Prescription." Here we present a summary of the discussion, including knowledge gaps, areas of controversy, and priorities for research. The conference agenda, discussion questions, and plenary session presentations are available on the KDIGO website: http://kdigo.org/conferences/ controversies-conference-on-dialysis-initiation/. It should be noted that patients who have a clear path to preemptive or planned kidney transplantation were not considered by the discussants.

\section{DIALYSIS MODALITIES AND AVAILABILITY}

Dialysis modalities include in-center, satellite or self-care, where dialysis is undertaken with access to support staff to aid in the dialytic procedure, and home hemodialysis, as well as continuous ambulatory and automated peritoneal dialysis. Prescription patterns can be categorized as conventional, incremental, intensive (short daily or nocturnal), trial-based, and palliative. Availability of modalities and prescription patterns is usually more a function of local resources, reimbursement policies, and infrastructure than informed patient preferences. In some parts of the world, in-center hemodialysis is the predominant modality, whereas a "peritoneal dialysis first" approach is taken in a number of jurisdictions with excellent outcomes. In industrialized countries, peritoneal dialysis is often more cost-effective than hemodialysis, yet the opposite may be true for countries with no local manufacturing of peritoneal dialysis fluids or with tariffs on importing peritoneal dialysis supplies. ${ }^{19-21}$ Factors that have been independently associated with a lower likelihood of use of peritoneal dialysis are diabetes as the cause of end-stage kidney disease (ESKD), higher health care expenditure as a percent of gross domestic product, a larger number of private, for-profit hemodialysis facilities, and greater cost of peritoneal dialysis consumables relative to staffing. ${ }^{20}$
Early mortality (death within the first 90 days of starting dialysis) disproportionately affects patients receiving in-center hemodialysis. This is likely due, at least in part, to selection bias because patients with acute kidney injury complicating chronic kidney failure or those with poorer health status are more likely to use in-center hemodialysis than peritoneal dialysis. ${ }^{22}$ Strategies to reduce early mortality are not well studied.

The only absolute contraindication for maintenance hemodialysis is the absence of possible vascular access or prohibitive cardiovascular instability. Peritoneal dialysis is contraindicated if the peritoneal cavity is obliterated, the membrane is not functional, or catheter access is not possible. Anuria is not a contraindication for peritoneal dialysis. All other health conditions are relative contraindications, and therefore the selection of dialysis modality needs to reflect informed patient choice with decision support appropriate to the health care system. Patients and caregivers need to be informed of the challenges, considerations, and trade-offs of the different dialysis modalities so that modality selection can be tailored to their health and social circumstances.

In multiple countries it has been reported that men more commonly receive dialysis than do women. ${ }^{2,23,24}$ Further investigation is needed to clarify whether and where disparities exist, and whether disparities result from biological differences or sociocultural biases.

Of note, conference participants recognized that preserving residual kidney function is important and should be a goal for all clinicians and dialysis patients. Yet residual kidney function should not be the sole consideration in selecting the initial dialysis modality, because the quality of evidence comparing decline in residual kidney function across modalities is based on small, mostly single-center, observational studies from more than 2 decades ago. ${ }^{25}$ As such, available data are not robust enough to suggest one modality is favorable over another.

Similarly, although there is evidence that some patients may benefit from incremental versus thrice-weekly hemodialysis in terms of preserving residual kidney function, ${ }^{26}$ there is currently not enough evidence for widespread adoption of incremental dialysis as a means to preserve residual kidney function.

\section{Urgent versus nonurgent and planned versus unplanned starts}

Urgent starts are defined as those in which dialysis must be initiated imminently or in less than 48 hours after presentation to correct life-threatening manifestations. Nonurgent starts are those in which dialysis initiation can be more than 48 hours after presentation. A planned approach is one in which the modality has been chosen prior to the need for dialysis and there is an access ready for use at the initiation of dialysis. An unplanned start is dialysis initiation when access is not ready for use or requires hospitalization or when dialysis is initiated with a modality that is not the patient's choice. Hemodialysis or peritoneal dialysis is possible in both planned or unplanned and urgent or nonurgent start 
situations. However, patients who require urgent dialysis in the setting of hyperkalemia, volume overload, or marked uremia are not good candidates for urgent-start peritoneal dialysis. The following are 5 key elements to a successful urgent start in patients in whom peritoneal dialysis is considered by the physician and patient as optimal therapy: ${ }^{27}$

(i) Ability to place a peritoneal catheter within 48 hours

(ii) Staff education regarding use of catheter immediately after placement

(iii) Administrative support in inpatient and outpatient settings

(iv) Identification of appropriate candidates for urgent-start peritoneal dialysis

(v) Utilization of protocols in every step of the urgent-start process (from patient selection for peritoneal dialysis through appropriate post-discharge follow-up)

The major barriers to an urgent-start peritoneal dialysis program are lack of operators who can place a peritoneal dialysis catheter within the urgent start time frame (i.e., 48 hours) and limited capacity of the health care facility to support peritoneal dialysis treatment for urgent-start patients and train patients on short notice. Where technical expertise in PD catheter placement is lacking, this can generally be addressed by increasing access for training in interventional nephrology by nephrologists and/or radiologists. When critical illness, time, or capacity to offer hemodialysis and peritoneal dialysis limit the initial choice, patients need to subsequently be provided with support to enable transition to their preferred modality when feasible.

\section{PATIENT EDUCATION AND SUPPORT}

\section{Preparation for dialysis}

Education and decision aids are essential in helping patients to better understand kidney failure, weigh available treatments, maintain a feeling of control, and share information with family members and/or carers. ${ }^{14}$ Additionally, early education is associated with lower mortality after dialysis is initiated. ${ }^{15}$

Effective education is usually offered to patients as they approach CKD G4. ${ }^{28}$ Education materials may include tours or videos with interviews of patients using the different modalities. In the absence of patient- or system-specific contraindications to a form of dialytic therapy, all options should be equally represented. Comprehensive education may also be available in the inpatient setting and for those who did not have regular follow-up with a nephrologist or access to dialysis education prior to starting dialysis.

The dialysis modality is ideally chosen with timely and shared decision-making among the health care team, patients, and their carers. Discussions about options and implications of various dialysis modalities need to include persons who start dialysis in an unplanned fashion. The approach for choosing modality is ideally person-centered, engaging the patient in choosing the dialysis modality in the context of their goals for care, local resources, out-of-pocket costs, capacities of regional health care facilities, and medical feasibility.
Table 1 | Patient and health care system barriers to homebased or self-care dialysis and potential solutions

\begin{tabular}{|c|c|}
\hline Barriers & Potential solutions \\
\hline Patient/carer-specific & Patient \& carer support \\
\hline Lack of awareness & $\begin{array}{c}\text { Education and training: flexible } \\
\text { group and individualized } \\
\text { training programs }\end{array}$ \\
\hline \multirow[t]{2}{*}{ Physical and cognitive barriers } & $\begin{array}{l}\text { Carer programs: increased } \\
\text { support and peer education }\end{array}$ \\
\hline & $\begin{array}{l}\text { Home visits, assisted home } \\
\text { dialysis, remote monitoring } \\
\text { Provision of dialysis in } \\
\text { nursing homes }\end{array}$ \\
\hline Social considerations & $\begin{array}{l}\text { Government policy and } \\
\text { incentive programs }\end{array}$ \\
\hline Carer burden & $\begin{array}{l}\text { Transparent information } \\
\text { regarding burden of dialysis } \\
\text { Respite care for carers }\end{array}$ \\
\hline Out-of-pocket costs & $\begin{array}{l}\text { Public policies to eliminate } \\
\text { or minimize out-of-pocket costs } \\
\text { Reimbursement of out of } \\
\text { pocket costs }\end{array}$ \\
\hline Physical space at home & $\begin{array}{l}\text { Independent community homes } \\
\text { Development in technology }\end{array}$ \\
\hline $\begin{array}{l}\text { Perceptions and fears of } \\
\text { dialyzing at home (e.g., } \\
\text { needle phobia, body image) }\end{array}$ & $\begin{array}{l}\text { Education and counseling: } \\
\text { psychosocial therapy }\end{array}$ \\
\hline Accessibility & Public advocacy \\
\hline By patient location & Independent community houses \\
\hline $\begin{array}{l}\text { Distance between patient's } \\
\text { home and training center }\end{array}$ & Home training programs \\
\hline $\begin{array}{l}\text { Extended care facilities } \\
\text { Lack of infrastructure }\end{array}$ & Flexible and individualized programs \\
\hline Delivery models & $\begin{array}{l}\text { Innovations: hub \& spoke, } \\
\text { supportive networks, centers } \\
\text { of excellence }\end{array}$ \\
\hline Economic & Incentive payments \\
\hline Within-health care variability & \\
\hline Health care team bias & Health care team training \\
\hline
\end{tabular}

Patients do perceive that home dialysis (peritoneal dialysis or hemodialysis) offers the opportunity to thrive; improves freedom, flexibility and well-being; and strengthens relationships. ${ }^{8,13}$ With appropriate adequate predialysis support and training, it is estimated that up to $50 \%$ of patients with ESKD will attain self-care dialysis. ${ }^{29}$ However, some voice anxiety and fear about performing dialysis treatments at home because of lack of confidence in their ability to master the technical aspects of dialysis, including self-cannulation for home hemodialysis, and because of isolation from medical and social support. ${ }^{8,13}$ Because many more patients around the world could be dialyzing at home or undertaking self-care than are doing so presently, the conference attendees recommended a goal of encouraging and supporting patients to select a home-based therapy (peritoneal dialysis or home hemodialysis) or self-care dialysis and to identify ways of overcoming barriers to this goal (Table 1). At the same time, it was recognized that many patients in many parts of the world will need or prefer in-center hemodialysis and that available dialysis modalities in some countries may depend upon local circumstances. 


\section{Supporting patients during dialysis}

After a patient starts dialysis, the health care team needs to provide ongoing support to optimize the health benefits of the selected modality. Support may be needed most in the period immediately after initiation and may decrease over time. Early attrition from peritoneal dialysis or home hemodialysis may result from catheter or mechanical problems, infection, management in a small center, or delayed referral to nephrologists or for CKD care. ${ }^{30}$ Anticipating and preventing these modifiable factors or quickly addressing such issues is important. Patient confidence in handling home dialysis procedures needs to be assessed prior to the start of dialysis and subsequent to initiation.

Frailty can impact dialysis complications and overall patient experience as well as prognosis. Because frailty can occur at any age, it is ideally assessed on a regular basis so that any reversible issues are identified and used to inform decisionmaking about treatment and support for the patient.

\section{TIMING AND PREPARATION FOR DIALYSIS INITIATION}

A specific estimated glomerular filtration rate (eGFR) value for initiating dialysis in the absence of symptomatic kidney failure has not been established. Indeed, the IDEAL study did not demonstrate any clinical benefit in commencing dialysis at higher levels of eGFR, and the variability in measurement of eGFR in CKD G5 is such that it should not be considered to reliably reflect kidney function. ${ }^{31}$ Registry data indicate mean predialysis eGFR varies among countries (approximately $5 \mathrm{ml} / \mathrm{min}$ per $1.73 \mathrm{~m}^{2}$ in Taiwan; average 8.5 in the UK, 7.3 in Australia, 6.4 in New Zealand, 9-10 in Canada and France, and 11 in the US). ${ }^{32-35}$ Generally, current guidelines do not support preemptive dialysis initiation, ${ }^{36-38}$ although an exception is the 2011 European guideline. ${ }^{39}$ While the optimal timing for starting dialysis is unclear, and in clinical practice the reasons for initiating dialysis are varied, ${ }^{40}$ risk equations can be helpful in predicting a time frame for when kidney replacement therapy may be necessary (Table 2).

Initiation of dialysis is usually considered when one or more of the following are present: symptoms or signs attributable to kidney failure (e.g., neurological signs and symptoms attributable to uremia, pericarditis, anorexia, medically resistant acid-base or electrolyte abnormalities, reduced energy level, weight loss with no other potential explanation, intractable pruritus, or bleeding); inability to control volume status or blood pressure; and a progressive deterioration in nutritional status refractory to interventions. ${ }^{36}$ Depending on the patient's preferences and circumstances, an aggressive trial of medical nondialytic management of advanced CKD symptoms may be warranted before initiating maintenance dialysis.

In adults older than 60 years, in the absence of acute kidney injury and where low levels of albuminuria exist, declines in eGFR may be relatively slow, ${ }^{34}$ and therefore the risk of dying before the need to initiate dialysis is higher than in other populations. Indeed, $20 \%$ to $35 \%$ of older CKD G4-G5 patients die per year before reaching dialysis. ${ }^{41}$ Additionally,
Table 2 | Risk equations for predicting time frame to needing kidney replacement therapy

\begin{tabular}{ccc}
\hline Patient group & Risk predicted & Reference \\
\hline CKD G3 or G4 & $\begin{array}{c}\text { 5-year kidney replacement } \\
\text { therapy predicted by age, } \\
\text { sex, eGFR, hemoglobin, }\end{array}$ & $\begin{array}{c}\text { Schroeder } \\
\text { et al. }\end{array}$ \\
& $\begin{array}{c}\text { proteinuria/albuminuria, systolic } \\
\text { BP, antihypertensive medication } \\
\text { use, and diabetes and } \\
\text { its complications }\end{array}$ &
\end{tabular}

CKD in older adults

5 -year mortality predicted by

Bansal et al. ${ }^{44}$ age, sex, race, eGFR, urine albumin-to-creatinine ratio, smoking, diabetes mellitus, and history of heart failure and stroke

$>75$ years old, within 3 months of dialysis

Mortality predicted by age, gender, specific comorbidities, albumin levels, and mobility

$>15$ years old initiating dialysis

6-month mortality predicted by older age, underweight, chronic lung disease, coronary artery disease, peripheral

vascular disease, cerebrovascular disease (particularly for

patients $<60$ years of age), late referral to nephrologist care, and underlying cause of kidney disease.

BP, blood pressure; CKD, chronic kidney disease; eGFR, estimated glomerular filtration rate.

older patients have a lower likelihood of survival in the 90 days after initiating dialysis. ${ }^{42}$ If patients have no other indications for starting dialysis, the decision may be made to delay initiation of dialysis in older patients until eGFR falls to $<6 \mathrm{ml} / \mathrm{min}$ per $1.73 \mathrm{~m}^{2}$. In patients with late-stage $\mathrm{CKD}$, it is important to discuss options for both medical and dialytic management in the event that acute kidney injury or additional significant illness occurs. Decision-making, including advanced care planning, can be aided using predictive models such as those developed by Couchoud et al. ${ }^{43}$ Bansal et al., ${ }^{44}$ and Ivory et al. ${ }^{45}$ This planning often includes individualized discussions regarding clinical course, goals of therapy, and patient preferences. Patients need to be made aware of the options of medical management without dialysis, such as supportive care and/or comfort measures and hospice care, when appropriate. ${ }^{4-48}$

\section{Predialysis assessments}

For patient-reported outcome measures, available assessments pertain to symptoms, objective markers of nutrition, functional capacity, and markers of kidney function. Available clinical reported outcomes include muscle strength, gait speed, body mass index, and biomarkers such as eGFR, serum albumin, etc. There is considerable variation in physician reliance on biochemical and hematologic variables such as creatinine, eGFR, urea, bicarbonate, potassium and phosphate levels, and hemoglobin levels among patients at the start of dialysis. ${ }^{40}$ Indices that measure frailty in CKD may be useful for informing patient decision-making, ${ }^{49}$ but further 
Table 3 | Dialysis modality and initiation timing: research needs and proposals

Key questions and approaches for investigation

\section{Dialysis initiation \\ Whether to initiate}

When to initiate

Timing of referrals and unplanned starts

\section{Choice of modality}

\section{Dialysis prescription}

Monitoring
- Can a CKD Frailty Index be used to inform patient decision-making? What would constitute the index-could it be based on the IPOS-Renal index?

- Could a CKD Frailty Index be combined with traditional and novel biomarkers and clinical scoring systems (serial assessments of fluid status, nutritional status and/or body composition) to guide initiation of dialysis?

- To what extent do uremic symptoms change after initiation of dialysis?

- Could a CKD Frailty Index be used to identify clinically important changes over time in individuals before dialysis and after initiation of dialysis? Are the changes different with HD versus PD?

- Is it possible to predict which patients improve and which get worse?

- With aggressive medical management, can the initiation of dialysis be delayed safely?

- Can an integrated care model improve quality and decrease costs for patients with kidney disease as they transition from CKD G5 to G5D (https://innovation.cms.gov/initiatives/comprehensive-esrd-care/)?

- How can the number of unplanned starts be reduced?

- How can referral and optimal management of patients with advanced CKD be improved?

- How can CKD patients at highest risk of acute kidney injury or heart failure exacerbations be identified?

- Do tablet holidays from drugs such as diuretics, ACE inhibitors, metformin, and NSAIDs prevent exacerbations?

- How can outcomes for post-acute kidney injury patients be improved?

- What measures can promote kidney recovery among acute kidney injury patients (in inpatient and outpatient settings)?

- Should dialysis be different for CKD G5 to G5D versus CKD with superimposed acute kidney injury?

- What is the real-world effectiveness and cost effectiveness of universal screening for CKD in high-risk populations?

- How effective are public health surveillance systems (public health lab and provider prompts) for CKD screening and risk prediction?

- Can multidisciplinary care during transition periods improve patient outcomes such as survival, hospitalization, cost-effectiveness, and quality of life?

- For patients choosing PD with a late referral, how do the outcomes compare for those who start with PD versus those who start with a short period of HD?

- What are the outcomes of urgent start PD versus short-term and long-term HD as evaluated in large-scale studies?

- What is the preferred timing for educating patients regarding dialysis modalities? Does the optimal time vary based on patient characteristics?

- What is the optimal content and format for educating patients regarding the advantages and disadvantages of each modality? How do we check their understanding?

- What are the outcomes of various dialysis modalities in subgroups of patients (e.g., pediatric, pregnant)?

- What are the characteristics and commonalities of "unexpected" deaths within the first 90 days of initiating dialysis, particularly in those with low burden of coexisting illnesses?

- What are the barriers to equal gender access to therapies, and how do these vary by country?

- Is respite care effective in retaining patients on home dialysis?

- What is the effectiveness of telemedicine and/or remote monitoring compared with conventional care in patients undergoing home dialysis to increase time on therapy and reduce complications?

- What is the threshold of kidney function or related symptoms at which to consider incremental dialysis?

- As evaluated by randomized controlled trials, how do outcomes such as residual kidney function and patient-reported outcomes compare with incremental dialysis versus conventional, full-dose initiation (HD or PD)?

- How does telemedicine and/or remote monitoring compare with conventional care in patients undergoing home dialysis?

ACE, angiotensin-converting enzyme; CKD, chronic kidney disease; HD, hemodialysis; IPOS, Integrated Palliative care Outcome Score; NSAID, nonsteroidal anti-inflammatory drug; PD, peritoneal dialysis; RCT, randomized controlled trial.

research is needed regarding the effectiveness of this approach (Table 3).

The timing of predialysis assessments depends on the absolute level and rate of decline in kidney function, symptom load, and associated metabolic, hematologic, and clinical comorbidities but will generally fall within the range of monthly to every 3 months. There was general consensus that symptoms such as anorexia, nausea, and fatigue should be improved or resolved within 3 months after starting dialysis therapy, although it was recognized that there is a lack of data to inform interpretation of symptom changes after initiation of kidney replacement therapy.

\section{Predialysis care and referrals}

Timely predialysis care has been associated with improvement in measurable outcomes such as delayed initiation of dialysis, cardiovascular complications, and mortality. ${ }^{50}$ Optimal multidisciplinary predialysis care includes not only timely referral but also frequent visits where patients can access different members of the care team. ${ }^{51}$ Registry data report wide variation in the transition period from CKD G5 to CKD G5D. A minimum 90-day transition period aligns with acute kidney injury recovery, registries, and early mortality on dialysis; however, the effects of interventions on lifestyle and risk factor modification may require years to take effect. The 
Table 4 | Strategies to avoid late referral for specialized nephrology care

\begin{tabular}{ll}
\hline Provider & \multicolumn{1}{c}{ Strategy } \\
\hline Primary care & - Education \\
& o Recognizing at-risk groups \\
& o Screening (eGFR, UACR, risk prediction) \\
& o Preventive treatment \\
& o Timely referrals \\
Laboratory & - Improved availability of eGFR reporting \\
& and uACR testing \\
& - Automated risk reporting and prompts for referral \\
& - Decision support integrated into EMR \\
Public health & Predefined multi-component CKD screen test \\
& Regional reporting of geographic and \\
& demographic trends in CKD screening and diagnosis \\
& Designation of CKD as a reportable disease \\
& when risk threshold is reached \\
& Early notification surveillance systems for \\
& patients and primary care providers \\
& - EMR notification system of need for screening \\
& - Evaluation of cost-benefit of mass screening \\
& Public awareness campaign \\
\hline
\end{tabular}

CKD, chronic kidney disease; eGFR, estimated glomerular filtration rate; EMR, electronic medical records; $\mathrm{UACR}$, urine albumin-to-creatinine ratio.

predialysis CKD care time frame is ideally long enough to encompass the 90-day transition period.

Recommendations for "timely referral" to a nephrologist, particularly for access creation, are mostly based on time to dialysis-for example, 6 months before the need to start dialysis. Although the timing to start dialysis can be difficult to accurately predict, evidence-based kidney failure risk equations could be used to create a more standardized approach. ${ }^{52}$ However, this must be combined with, rather than replace, clinical judgment. Strategies to avoid delayed referral are listed in Table 4.

\section{Tailoring timing and support for certain subgroups of patients}

Initiation of dialysis in the setting of a failing graft or moving between dialysis modalities. Patients with a failing kidney transplant may not be adequately prepared for approaching ESKD because the focus of care may be to maintain graft function rather than prepare for dialysis. CVCs are used in nearly two-thirds of patients with failed kidney transplant grafts, ${ }^{53}$ and the relatively low prevalence of AVFs or AVGs in this group at initiation of dialysis needs to be investigated more thoroughly. ${ }^{53}$ Collaboration with CKD programs may be beneficial, especially as eGFR declines below 20 to $30 \mathrm{ml} /$ min per $1.73 \mathrm{~m}^{2}$. Education and review of patient preferences and life goals are important, as is the preparation for possible next steps such as hemodialysis, peritoneal dialysis, another kidney transplant, or supportive care, as part of the patients' ESKD life plan. ${ }^{54}$ Studies based on global kidney transplant registries will be needed to track specific issues in managing patients with failing kidney grafts.

Data from the United States indicate that peritoneal dialysis patients under nephrologist supervision have a very low rate of arteriovenous access upon transition to hemodialysis. ${ }^{55}$ Hence, time-dependent surveillance of effective clearances and ultrafiltration volumes are necessary in patients on PD. Predictive models are needed to identify peritoneal dialysis patients that require transition preparation such as education and vascular access creation, ${ }^{56,57}$ particularly to support home hemodialysis when appropriate.

Pediatric initiation of dialysis. Pediatric models of care were not a focus of this conference. In children, the unique aspects of growth, nutrition, and cognitive as well as emotional maturation increase the complexity of diagnosis, treatment, and decision-making, and therefore having a multidisciplinary team to address these issues is especially important. Children will have different needs as they age, and it is important to recognize that young adulthood and the transition to independent living can be a time when patients need a lot of support. In the pediatric population, CKD is more likely to lead to ESKD versus death, and therefore, validated prediction models for referral to preemptive transplant or dialysis are especially important. In a retrospective cohort study of 603 children with eGFR $<60 \mathrm{ml} / \mathrm{min}$ per $1.73 \mathrm{~m}^{2}$, kidney failure risk equations provided excellent discrimination of the risk of developing ESKD in 1 or 2 years in those with a kidney failure risk equation (KFRE) score of at least $13.2 \%$ compared with those with a score less than $13.2 \% .{ }^{58}$ A pediatric global initiative to determine the impact of early versus late initiation of preemptive transplant or dialysis could be conducted as a pragmatic randomized controlled trial, with a similar approach to that of the IDEAL study in adults ${ }^{59}$ but with outcomes concentrated on growth, cognitive development, and nutritional status.

Pregnancy and initiation of dialysis. The definitive method for determining pregnancy in the setting of kidney failure is ultrasound, because levels of $\beta$-hCG can be increased during kidney failure, leading to false-positive pregnancy test results. ${ }^{60}$ Conception pre-dialysis results in higher infant survival and lower likelihood of prematurity than conception after starting dialysis. ${ }^{61}$ Cohort data have indicated that dialysis intensity affects outcomes, with longer durations resulting in a higher live birth rate, longer gestational age, and greater infant birth weight. ${ }^{62}$ In pregnant women undergoing dialysis, an intensive prescription should be considered. During pregnancy, women on dialysis need to be monitored for low potassium, phosphate, and folate, as well as high glucose, especially for patients undergoing tidal peritoneal dialysis. It is also important to evaluate for anemia and assess nutrition and magnesium levels. Previously the timing of initiation of dialysis in pregnancy was based on high urea levels (blood urea nitrogen $[\mathrm{BUN}]>36 \mathrm{mmol} / \mathrm{l}$, goal to decrease $<18 \mathrm{mmol} / \mathrm{l}$ ). The goal currently is to initiate at BUN $<18 \mathrm{mmol} / \mathrm{l}^{63}$ also recognizing anecdotally the importance of fluid, electrolyte, and acid-base disturbances. A possible strategy for research is evaluating registry data to determine the impact of early versus late initiation of dialysis in pregnancy, along the lines of the IDEAL study in adults ${ }^{59}$ but with outcomes concentrated on live birth rate, pregnancy survival, gestational age, and birth weight. 
Table 5 | The integrated ESKD life plan approach for dialysis access implementation $^{\text {a }}$

Options for dialysis access according to ESKD life plan

- Conservative treatment (no dialysis, no access)

- Pre-emptive transplant (no dialysis access; consider in future as needed)

- Peritoneal dialysis (PD catheter, no AV access; consider in future as needed)

- Hemodialysis (AVF, AVG, CVC: access planning)

Hemodialysis access planning

- Consider likelihood of long-term survival (> 1 year)

o Poor: Maintain conservative care and periodically reassess for changes. These patients are more likely candidates for AVG or CVC o Good: Assess quality of vessels for AV access

- AV access not feasible $\rightarrow$ CVC

- AV access feasible $\rightarrow$ consider AVF likelihood of usability success; decision on the preferred access is to achieve complication-free access while preserving vessels for future sites per individualized ESKD life plan: e.g., in an optimal scenario where all vessels available: forearm AVF, upper arm AVF, AVG

o Once $\mathrm{HD}$ access is established, consider improvements of care

- Timely shift to kidney transplant or PD, whenever feasible and appropriate

- Shift from CVC to AVF or AVG, as soon as possible when feasible and appropriate

- Secondary shift from AVG to AVF, when AVG is failing; when feasible and appropriate

o When planning a dialysis access procedure, always keep in mind next possible access(es) for the individual patient to attain the longest and most feasible access life plan for the individual patient based on their ESKD life plan and goals of care

$A V$, arteriovenous; $A V F$, arteriovenous fistula; $A V G$, arteriovenous graft; $C V C$, central venous catheter; ESKD, end-stage kidney disease; PD, peritoneal dialysis.

a Modified in accordance to the KDOQI Vascular Access Guidelines 2018, presented at NKF SCM 2018, Austin, TX (April 2018).

\section{DIALYSIS ACCESS AND PREPARATION}

It is widely accepted that preemptively establishing dialysis access leads to better patient outcomes; however, there are significant challenges and barriers within health care systems and among payers and patients to establish dialysis access that is matured and functional for dialysis initiation (Supplementary Table S1). ${ }^{64-70}$ According to most guidelines, peritoneal dialysis access should be prepared whenever possible at least 2 weeks before starting kidney replacement therapy. ${ }^{71,72}$ For patients referred late, this recommendation might determine a temporary or definitive shift of patients to hemodialysis. However, recent evidence has demonstrated that urgent-start peritoneal dialysis is possible and safe, if properly conducted. ${ }^{73}$ Although meeting participants acknowledged the benefits of fistulas, ${ }^{74}$ they recognized the "fistula-first" approach is not appropriate for all patients. There is a need to reconsider established paradigms for dialysis access within the framework of the patient's ESKD life plan, ${ }^{54}$ taking into account individual patient and vessel characteristics and patient life goals and preferences. Age, comorbidities, likelihood of long-term survival, treatment goals, and timing of dialysis initiation are all factors that could affect the choice for access and require individualization for each patient. ${ }^{54,75}$ It is also important to keep in mind the subsequent best treatment modality and access for the individual patient (Table 5). Many patients are able to undertake home HD with permacaths, and the lack of surgically created vascular access is not an absolute contraindication to selfmanaged home HD.

\section{Selection and management of access as related to funding policies}

Selection and management of access that is tightly linked to funding policies may have serious implications for patient health. In resource-limited environments, if surgery is less expensive than endovascular procedures, it can put patients at risk for early exhaustion of vascular access sites. Economic constraints leading to prolonged use of nontunneled CVCs can increase the risk of infection for patients. Conversely, in resource-rich environments, well-reimbursed endovascular procedures can lead to over-intervention, vessel damage, and premature loss of vascular access. Ideally, financial incentives should be aligned with best care practices. Unfortunately, there is no consensus, evidence base, or outcome measure for best practices at the individual patient level that can be measured on a population basis. Additionally, specific outcomes used as a measure of best practice at the population level do not always apply at the patient level. To provide individualized patient care, it may be necessary to adopt "process" versus outcomes measures of best practice, such as percent of patients referred to and evaluated for vascular access before dialysis initiation. Targeting process best practices (e.g., referral for vascular access) may lead to better best practice outcomes (e.g., more functional AV accesses). Priorities for research and education are described in Supplementary Table S2.

\section{Access "exit strategies"}

Important in the decision-making process for choosing initial dialysis access is the consideration of what options are available if the initial access fails. Currently there is no published evidence to inform back-up, and therefore research is needed to evaluate the optimal order of exit strategies in access for hemodialysis. Given the various modalities and access needs of each patient, such access contingency and succession plans must be individualized. At best, the consequences of lack of planning — as discussed above for failing transplants and PD patients-highlights the need for access contingency and succession planning.

\section{PROVIDING "ADEQUATE" DIALYSIS AND SYMPTOM CONTROL}

For decades, dialysis adequacy has been defined by small solute clearance (Kt/V and urea reduction ratio in hemodialysis; $\mathrm{Kt} / \mathrm{V}$ and creatinine clearance in peritoneal dialysis). Measuring small solute clearance has been emphasized in clinical practice guidelines, used as the basis for clinical performance measures and/or payment, and treated by many clinicians as dogma. However, evidence for relationships between small solute clearance, plasma levels of these solutes, and clinical outcomes and/or symptomatology is weak. It is increasingly recognized that small solute clearance reflects only one of many aspects of dialysis care that are likely to 


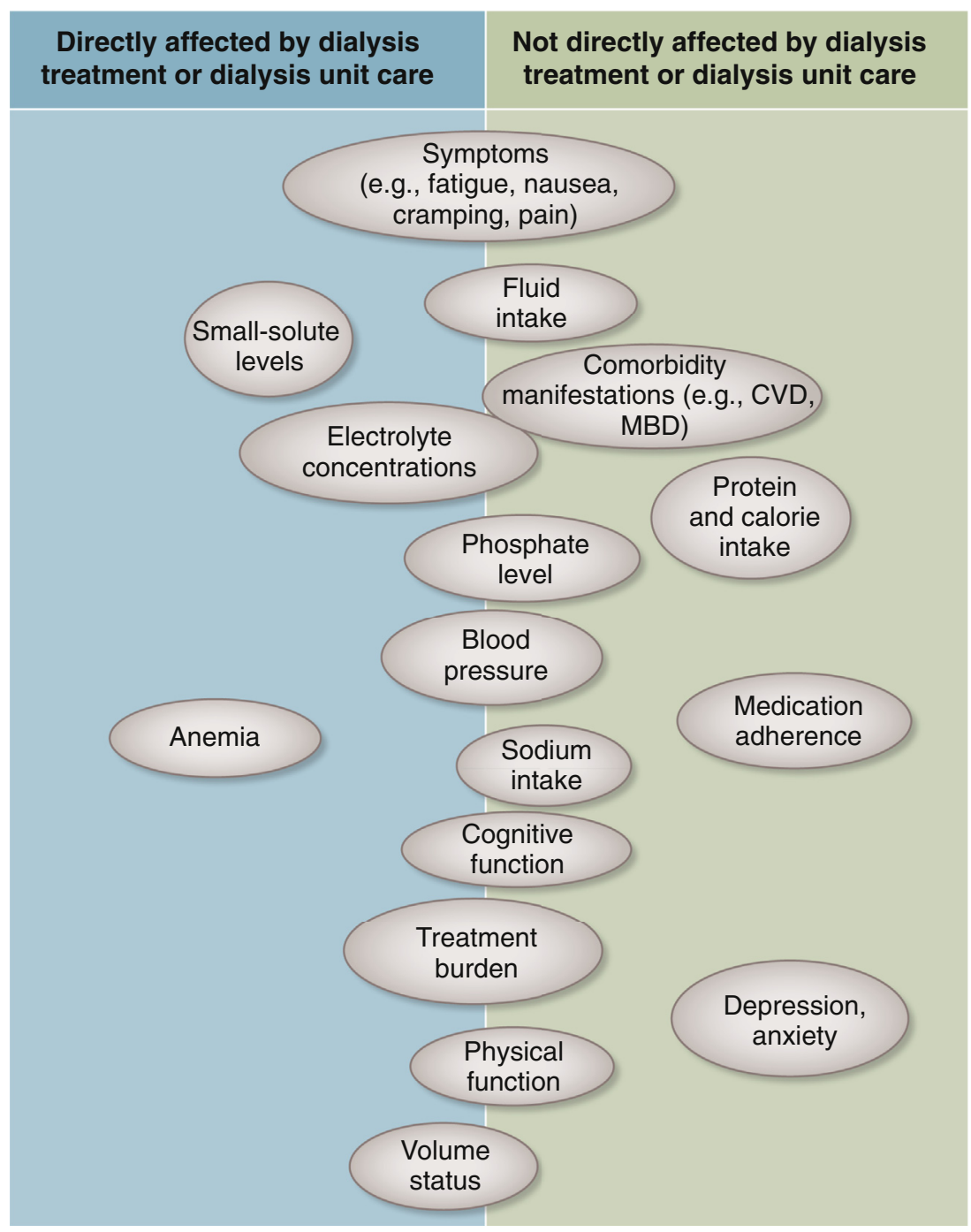

Figure 1 | Potential targets for goal-directed dialysis care. This schematic illustration serves to identify targets or outcomes that might be considered in developing goal-directed dialysis care. Some targets or outcomes, such as anemia, are principally affected by dialysis care providers through an erythropoiesis-stimulating agent and iron dosing, while others, such as protein and calorie intake, are less directly affected. Many targets or outcomes are affected by both dialysis treatment or care and by nondialysis factors. CVD, cardiovascular disease; $M B D$, mineral and bone disease.

affect outcomes. ${ }^{16,18,76}$ Therefore, solute kinetic goals should be interpreted and implemented in the context of the patient's overall goals and clinical status.

Meeting participants favored a comprehensive view of ESKD care with a more multifaceted paradigm replacing what is currently referred to as "dialysis adequacy." Multiple measures and goals should be considered when assessing adequacy of dialysis, including small solute clearance, residual kidney function, volume status, biochemical measures, nutritional status, cardiovascular function, symptoms, and the patient's experiences and goals. While many meeting participants felt that a rigid emphasis on solute clearance does not serve the interests of all patients, there was general agreement that clinicians should continue to recognize accepted minimums for small solute removal during dialysis. It was also recognized that research is needed to investigate the importance of other solutes including middle and large solutes, protein-bound and carbamylated molecules, and metabolic products of intestinal bacteria as potential contributors to poor functional status, symptoms, comorbidities, and mortality among patients being treated with maintenance dialysis.

It should be recognized that patients may interpret "adequacy" differently than clinicians, and therefore goals for treatment should be individualized and reassessed over time. To this end, many — but not all-meeting participants propose that the term "adequate dialysis" be changed to "goaldirected dialysis," which specifically refers to using shared decision-making between the patient and care team to establish realistic care goals to allow the patient to meet his or her own life goals and allow the clinician to provide individualized, high-quality dialytic care.

The components of goal-directed dialysis can be thought of as those directly affected by the dialysis procedure, such as small solute clearance, electrolyte concentrations, volume 


\section{Table 6| Implementation needs for goal-directed dialysis}

- Education for clinical care teams

- Tools for eliciting and documenting patient goals

- Incorporation of symptom assessment and other patient-centered outcomes into routine clinical practice

- Increased flexibility for dialysis schedules

- Establishment of metrics for success

- Quality assessment approaches that accommodate individualized targets

- Buy-in from multiple stakeholders

- Aligned incentives across stakeholders

status, and intra-dialytic symptoms, as well as those that are indirectly affected by the dialysis procedure, such as symptom burden, nutritional status, activity level, work capacity, and social engagement (Figure 1). Priorities should be individualized and consistent with achievable goals for the patient. A patient's priorities will likely change between the initial months of dialysis and thereafter; therefore, prioritization requires ongoing discussions between patients and clinicians about realistic expectations and prognosis. Discussion may need to be tailored depending on the patient's cognitive function, health literacy, numeracy, socioeconomic status, and initial dialysis experiences.

\section{Implementing goal-directed dialysis}

Implementing goal-based care would require a significant shift from current international practice as well as buy-in from multiple stakeholders including patients, providers, regulators, and payers, who may have conflicting expectations and motivations (Table 6). Tools are needed for facilitating communication between patients and clinicians because they may have limited experience expressing or eliciting, respectively, care objectives and priorities. In health care systems, incentives for providers and payers need to be aligned, and methods for assessing patient-centered outcomes such as symptoms will need to be validated and then incorporated into routine care, possibly through technology. As was discussed previously for vascular access, assessing the quality of highly individualized care will likely require the development of process measures.

\section{CONCLUSION}

A major theme identified during the conference was the need to move away from a "one-size-fits-all" approach to dialysis and provide more individualized or personalized care. Identifying and achieving patient-centered goals is now recognized as an important component of dialysis care, and these will require provider tools, patient tools, and alignment of goals and incentives among patients, caregivers, health care providers, regulators, and payers. Meeting participants acknowledge that any suggestion for moving toward patientcentered, goal-directed dialysis assumes that whatever systems are in place for delivering dialysis are modifiable. Admittedly, in some regions around the world, ability to change existing structures may be limited. Thus, the approaches outlined here are meant to serve as strategies that may be implemented via a variety of tactics depending on the local environment.

This conference is first of a series of dialysis controversies meetings to help provide a blueprint for delivery of optimal contemporary kidney replacement therapy. Subsequent topics in this conference series will address management of dialysis complications, innovations in kidney replacement therapy, and blood pressure and volume management in ESKD.

\section{APPENDIX}

\section{Other conference participants}

Ali K. Abu-Alfa, Lebanon; Joanne M. Bargman, Canada; Anthony J. Bleyer, USA; Edwina A. Brown, UK; Andrew Davenport, UK; Simon J. Davies, UK; Frederic O. Finkelstein, USA; Jennifer E. Flythe, USA; Eric Goffin, Belgium; Thomas A. Golper, USA; Rafael Gómez, Colombia; Takayuki Hamano, Japan; Manfred Hecking, Austria; Olof Heimbürger, Sweden; Barnaby Hole, UK; Daljit K. Hothi, UK; T. Alp Ikizler, USA; Yoshitaka Isaka, Japan; Kunitoshi Iseki, Japan; Vivekanand Jha, India; Hideki Kawanishi, Japan; Peter G. Kerr, Australia; Paul Komenda, Canada; Csaba P. Kovesdy, USA; Ed Lacson, Jr, USA; Maurice Laville, France; Jung Pyo Lee, Korea; Edgar V. Lerma, USA; Nathan W. Levin, USA; Monika

Lichodziejewska-Niemierko, Poland; Adrian Liew, Singapore; Elizabeth Lindley, UK; Robert S. Lockridge, USA; Magdalena Madero, Mexico; Ziad A. Massy, France; Linda McCann, USA; Klemens B. Meyer, USA; Rachael L. Morton, Australia; Annie-Claire Nadeau-Fredette, Canada; Hirokazu Okada, Japan; Jose J. Perez, USA; Jeff Perl, Canada; Kevan R. Polkinghorne, Australia; Miguel C. Riella, Brazil; Bruce M. Robinson, USA; Michael V. Rocco, USA; Steven J. Rosansky, USA; Joris I. Rotmans, the Netherlands; María Fernanda Slon Roblero, Spain; Navdeep Tangri, Canada; Marcello Tonelli, Canada; Allison Tong, Australia; Yusuke Tsukamoto, Japan; Kriang Tungsanga, Thailand; Tushar J. Vachharajani, USA; Ismay N. van Loon, the Netherlands; Suzanne Watnick, USA; Daniel E. Weiner, USA; Martin Wilkie, UK; Elena Zakharova, Russian Federation

\section{DISCLOSURE}

CTC declared having received consultancy fees from Medtronic and NxStage; speaker honoraria from Fresenius; and research support from Medtronic. MG declared having received consultancy fees from B. Braun Medical and speaker honoraria from Medtronic. DCHH declared having received speaker honoraria from Otsuka Japan and research support from National Health and Medical Research Council. AY-MW declared having received speaker honoraria from Sanofi Renal; and research support from Otsuka and Sanofi Renal. DCW declared having received consultancy fees from Akebia Therapeutics, Amgen, AstraZeneca, Boehringer Ingelheim, GlaxoSmithKline, Janssen, and Vifor Fresenius; speaker honoraria from Amgen and Vifor Fresenius; and research support from AstraZeneca. WCW declared having received consultancy fees from Akebia Therapeutics, AMAG, Amgen, AstraZeneca, Bayer, Daichii-Sankyo, Relypsa, and ZS Pharma; speaker honoraria from FibroGen; and research support from National Institutes of Health. CAP declared having served on advisory boards of AstraZeneca, Janssen Cilag, and Vifor; and received speaker honoraria from AstraZeneca, Novartis, Sanofi, and Vifor. All the other authors declared no competing interests.

\section{ACKNOWLEDGMENTS}

The conference was sponsored by KDIGO and supported in part by unrestricted educational grants from Akebia Therapeutics, Amgen, AstraZeneca, Baxter, Boehringer Ingelheim, Fresenius Medical Care, Kaneka, NxStage, Relypsa, Roche, Rockwell Medical, and Vifor Fresenius Medical Care Renal Pharma. We thank Jennifer King, PhD, for assistance with manuscript preparation.

\section{SUPPLEMENTARY MATERIAL}

Table S1. Barriers to choosing dialysis access.

Table S2. Research questions and priorities for education in dialysis access.

Supplementary material is linked to the online version of the paper at www.kidney-international.org. 


\section{REFERENCES}

1. Thomas B, Wulf $S$, Bikbov B, et al. Maintenance dialysis throughout the world in years 1990 and 2010. J Am Soc Nephrol. 2015;26:2621-2633.

2. Liyanage $T$, Ninomiya $T$, Jha $V$, et al. Worldwide access to treatment for end-stage kidney disease: a systematic review. Lancet. 2015;385:19751982.

3. Locatelli F, Del Vecchio L, Pozzoni P, et al. Nephrology: main advances in the last 40 years. J Nephrol. 2006;19:6-11.

4. Robinson BM, Akizawa $\mathrm{T}$, Jager $\mathrm{KJ}$, et al. Factors affecting outcomes in patients reaching end-stage kidney disease worldwide: differences in access to renal replacement therapy, modality use, and haemodialysis practices. Lancet. 2016;388:294-306.

5. Foster BJ, Mitsnefes MM, Dahhou M, et al. Changes in excess mortality from end stage renal disease in the United States from 1995 to 2013. Clin J Am Soc Nephrol. 2018;13:91-99.

6. Hasegawa T, Bragg-Gresham JL, Yamazaki S, et al. Greater first-year survival on hemodialysis in facilities in which patients are provided earlier and more frequent pre-nephrology visits. Clin J Am Soc Nephrol. 2009;4:595-602.

7. Tennankore KK, Soroka SD, Kiberd BA. The impact of an "acute dialysis start" on the mortality attributed to the use of central venous catheters: a retrospective cohort study. BMC Nephrol. 2012;13:72.

8. Walker RC, Hanson CS, Palmer SC, et al. Patient and caregiver perspectives on home hemodialysis: a systematic review. Am J Kidney Dis. 2015;65:451-463.

9. Evangelidis N, Tong A, Manns B, et al. Developing a set of core outcomes for trials in hemodialysis: An international Delphi survey. Am J Kidney Dis. 2017;70:464-475.

10. Morton RL, Snelling P, Webster AC, et al. Dialysis modality preference of patients with CKD and family caregivers: a discrete-choice study. Am J Kidney Dis. 2012;60:102-111.

11. Ronco C, Mason G, Nayak Karopadi A, et al. Healthcare systems and chronic kidney disease: putting the patient in control. Nephrol Dial Transplant. 2014;29:958-963.

12. Dahlerus $C_{1}$ Quinn $M$, Messersmith $E_{1}$ et al. Patient perspectives on the choice of dialysis modality: results from the Empowering Patients on Choices for Renal Replacement Therapy (EPOCH-RRT) study. Am J Kidney Dis. 2016;68:901-910.

13. Morton RL, Tong A, Howard K, et al. The views of patients and carers in treatment decision making for chronic kidney disease: systematic review and thematic synthesis of qualitative studies. BMJ. 2010;340:C112.

14. Winterbottom AE, Gavaruzzi T, Mooney A, et al. Patient acceptability of the Yorkshire Dialysis Decision Aid (YoDDA) booklet: a prospective nonrandomized comparison study across 6 predialysis services. Perit Dial Int. 2016:36:374-381.

15. Lacson E Jr, Wang W, DeVries C, et al. Effects of a nationwide predialysis educational program on modality choice, vascular access, and patient outcomes. Am J Kidney Dis. 2011;58:235-242.

16. Manera KE, Tong A, Craig JC, et al. Standardized Outcomes in Nephrology-Peritoneal Dialysis (SONG-PD): study protocol for establishing a core outcome set in PD. Perit Dial Int. 2017:37:639-647.

17. Finkelstein FO, Finkelstein $\mathrm{SH}$. Time to rethink our approach to patientreported outcome measures for ESRD. Clin J Am Soc Nephrol. 2017;12: 1885-1888.

18. Perl J, Dember LM, Bargman JM, et al. The use of a multidimensional measure of dialysis adequacy-Moving beyond small solute kinetics. Clin J Am Soc Nephrol. 2017;12:839-847.

19. Pike $E$, Hamidi V, Ringerike $T$, et al. More use of peritoneal dialysis gives significant savings: A systematic review and health economic decision model. J Clin Med Res. 2017:9:104-116.

20. van de Luijtgaarden MW, Jager KJ, Stel VS, et al. Global differences in dialysis modality mix: the role of patient characteristics, macroeconomics and renal service indicators. Nephrol Dial Transplant. 2013;28:1264-1275.

21. Karopadi AN, Mason G, Rettore E, et al. Cost of peritoneal dialysis and haemodialysis across the world. Nephrol Dial Transplant. 2013;28:25532569.

22. Jaar BG. The Achilles heel of mortality risk by dialysis modality is selection bias. J Am Soc Nephrol. 2011:22:1398-1400.

23. Piccoli GB, Alrukhaimi M, Liu ZH, et al. Women and kidney disease: reflections on World Kidney Day 2018: kidney health and women's health: a case for optimizing outcomes for present and future generations. Nephrol Dial Transplant. 2018:33:189-193.
24. United States Renal Data System. Annual Data Report 2017: End-stage Renal Disease in the United States: Chapter 1: Incidence, Prevalence, Patient Characteristics, and Treatment Modality. Available at: https:// www.usrds.org/2017/download/v2_c01_IncPrev_17.pdf. Accessed February 13, 2018.

25. Kjaergaard KD, Jensen JD, Peters $C D$, et al. Preserving residual renal function in dialysis patients: an update on evidence to assist clinical decision making. NDT Plus. 2011;4:225-230.

26. Mathew AT, Obi Y, Rhee CM, et al. Incremental dialysis for preserving residual kidney function-Does one size fit all when initiating dialysis? Semin Dial. 2018;31:343-352.

27. Ghaffari A. Urgent-start peritoneal dialysis: a quality improvement report. Am J Kidney Dis. 2012;59:400-408.

28. Levin A, Stevens PA, Bilous RW, et al. Kidney Disease: Improving Global Outcomes (KDIGO) CKD work group. KDIGO 2012 clinical practice quidelines for the evaluation and managment of chronic kidney disease. Kidney Int Suppl. 2013;3:1-150.

29. Goovaerts T, Jadoul M, Goffin E. Influence of a pre-dialysis education programme (PDEP) on the mode of renal replacement therapy. Nephrol Dial Transplant. 2005:20:1842-1847.

30. See EJ, Johnson DW, Hawley CM, et al. Risk predictors and causes of technique failure within the first year of peritoneal dialysis: an Australia and New Zealand Dialysis and Transplant Registry (ANZDATA) study. Am J Kidney Dis. 2018;72:188-197.

31. Wong MG, Pollock CA, Cooper BA, et al. Association between GFR estimated by multiple methods at dialysis commencement and patient survival. Clin J Am Soc Nephrol. 2014:9:135-142.

32. Sood MM, Manns B, Dart A, et al. Variation in the level of eGFR at dialysis initiation across dialysis facilities and geographic regions. Clin J Am Soc Nephrol. 2014;9:1747-1756.

33. Gilg J, Pruthi R, Fogarty D. UK Renal Registry 17th Annual Report: Chapter 1 UK Renal Replacement Therapy Incidence in 2013: National and Centre-specific Analyses. Nephron. 2015;129(Suppl 1):1-29.

34. United States Renal Data System. Annual Data Report 2017: Chronic Kidney Disease (CKD) in the United States: Chapter 8: Transition of Care in Chronic Kidney Disease. Available at: https://www.usrds.org/2017/ view/v1_08.aspx. Accessed February 8, 2018.

35. ANZDATA Registry. 39th Report, Chapter 1: Incidence of End Stage Kidney Disease. Adelaide, Australia: Australia and New Zealand Dialysis and Transplant Registry; 2017. http://www.anzdata.org.au.

36. National Kidney Foundation. KDOOI Clinical Practice Guideline for Hemodialysis Adequacy: 2015 update. Am J Kidney Dis. 2015;66:884-930.

37. Nesrallah GE, Mustafa RA, Clark WF, et al. Canadian Society of Nephrology 2014 clinical practice guideline for timing the initiation of chronic dialysis. CMAJ. 2014;186:112-117.

38. Watanabe $\mathrm{Y}$, Yamagata $\mathrm{K}$, Nishi S, et al. Japanese society for dialysis therapy clinical guideline for "hemodialysis initiation for maintenance hemodialysis. Ther Apher Dial. 2015;19(Suppl 1):93-107.

39. Tattersall J, Dekker F, Heimburger O, et al. When to start dialysis: updated guidance following publication of the Initiating Dialysis Early and Late (IDEAL) study. Nephrol Dial Transplant. 2011;26:2082-2086.

40. Heaf J, Petersons A, Vernere B, et al. Why do physicians prescribe dialysis? A prospective questionnaire study. PLoS One. 2017;12:e0188309.

41. United States Renal Data System. Annual Data Report 2017: End-stage Renal Disease in the United States: Chapter 5: Mortality. Available at: https://www.usrds.org/2017/view/v2 05.aspx. Accessed February 8, 2018

42. Chan KE, Maddux FW, Tolkoff-Rubin N, et al. Early outcomes among those initiating chronic dialysis in the United States. Clin J Am Soc Nephrol. 2011:6:2642-2649.

43. Couchoud CG, Beuscart JB, Aldigier JC, et al. Development of a risk stratification algorithm to improve patient-centered care and decision making for incident elderly patients with end-stage renal disease. Kidney Int. 2015;88:1178-1186.

44. Bansal N, Katz R, De Boer IH, et al. Development and validation of a model to predict 5-year risk of death without ESRD among older adults with CKD. Clin J Am Soc Nephrol. 2015:10:363-371.

45. Ivory SE, Polkinghorne KR, Khandakar Y, et al. Predicting 6-month mortality risk of patients commencing dialysis treatment for end-stage kidney disease. Nephrol Dial Transplant. 2017;32:1558-1565.

46. Davis JL, Davison SN. Hard choices, better outcomes: a review of shared decision-making and patient decision aids around dialysis initiation and 
conservative kidney management. Curr Opin Nephrol Hypertens. 2017;26: 205-213.

47. Sellars M, Clayton JM, Morton RL, et al. An interview sudy of patient and caregiver perspectives on advance care planning in ESRD. Am J Kidney Dis. 2018;71:216-224.

48. Ladin K, Pandya R, Kannam A, et al. Discussing conservative management with older patients with CKD: An interview study of nephrologists. Am J Kidney Dis. 2018;71:627-635.

49. van Loon IN, Goto NA, Boereboom FTJ, et al. Frailty screening tools for elderly patients incident to dialysis. Clin J Am Soc Nephrol. 2017;12:14801488.

50. Silver SA, Bell CM, Chertow GM, et al. Effectiveness of quality improvement strategies for the management of CKD: a meta-analysis. Clin J Am Soc Nephrol. 2017;12:1601-1614.

51. Ruggenenti P, Perticucci E, Cravedi P, et al. Role of remission clinics in the longitudinal treatment of CKD. J Am Soc Nephrol. 2008;19:1213-1224.

52. Tangri N, Grams ME, Levey AS, et al. Multinational assessment of accuracy of equations for predicting risk of kidney failure: A metaanalysis. JAMA. 2016;315:164-174.

53. Chan MR, Oza-Gajera B, Chapla K, et al. Initial vascular access type in patients with a failed renal transplant. Clin J Am Soc Nephrol. 2014;9: 1225-1231.

54. Woo K, Lok CE. New insights into dialysis vascular access: What is the optimal vascular access type and timing of access creation in CKD and dialysis patients? Clin J Am Soc Nephrol. 2016;11:1487-1494.

55. Pulliam J, Li NC, Maddux F, et al. First-year outcomes of incident peritoneal dialysis patients in the United States. Am J Kidney Dis. 2014;64:761-769.

56. Chiarelli G, Beaulieu M, Cozzolino M, et al. Vascular access planning in peritoneal dialysis patients. Perit Dial Int. 2008;28:585-590.

57. Gallieni M, Giordano A, Ricchiuto A, et al. Dialysis access: issues related to conversion from peritoneal dialysis to hemodialysis and vice versa. J Vasc Access. 2017;18(Suppl 1):41-46.

58. Winnicki E, McCulloch CE, Mitsnefes MM, et al. Use of the Kidney Failure Risk Equation to determine the risk of progression to end-stage renal disease in children with chronic kidney disease. JAMA Pediatr. 2018;172:174-180.

59. Cooper BA, Branley P, Bulfone $L$, et al. A randomized, controlled trial of early versus late initiation of dialysis. N Engl J Med. 2010;363:609-619.

60. Hou S. Pregnancy in chronic renal insufficiency and end-stage renal disease. Am J Kidney Dis. 1999;33:235-252.

61. Okundaye I, Abrinko P, Hou S. Registry of pregnancy in dialysis patients. Am J Kidney Dis. 1998;31:766-773.
62. Hladunewich MA, Hou S, Odutayo A, et al. Intensive hemodialysis associates with improved pregnancy outcomes: a Canadian and United States cohort comparison. J Am Soc Nephrol. 2014;25:1103-1109.

63. Asamiya Y, Otsubo S, Matsuda Y, et al. The importance of low blood urea nitrogen levels in pregnant patients undergoing hemodialysis to optimize birth weight and gestational age. Kidney Int. 2009;75:12171222.

64. Lopez-Vargas PA, Craig JC, Gallagher MP, et al. Barriers to timely arteriovenous fistula creation: a study of providers and patients. Am J Kidney Dis. 2011;57:873-882.

65. Donca IZ, Wish JB. Systemic barriers to optimal hemodialysis access. Semin Nephrol. 2012;32:519-529.

66. Lok CE, Oliver MJ, Su J, et al. Arteriovenous fistula outcomes in the era of the elderly dialysis population. Kidney Int. 2005;67:2462-2469.

67. Xi W, MacNab J, Lok CE, et al. Who should be referred for a fistula? A survey of nephrologists. Nephrol Dial Transplant. 2010;25:2644-2651.

68. Xi W, Harwood L, Diamant MJ, et al. Patient attitudes towards the arteriovenous fistula: a qualitative study on vascular access decision making. Nephrol Dial Transplant. 2011;26:3302-3308.

69. Chaudhry M, Bhola C, Joarder M, et al. Seeing eye to eye: the key to reducing catheter use. J Vasc Access. 2011;12:120-126.

70. Pisoni RL, Zepel L, Port FK, et al. Trends in US vascular access use, patient preferences, and related practices: An update from the US DOPPS Practice Monitor with international comparisons. Am J Kidney Dis. 2015;65:905-915.

71. Figueiredo A, Goh BL, Jenkins S, et al. Clinical practice guidelines for peritoneal access. Perit Dial Int. 2010;30:424-429.

72. Woodrow G, Fan SL, Reid C, et al. Renal Association Clinical Practice Guideline on peritoneal dialysis in adults and children. BMC Nephrol. 2017;18:333.

73. See EJ, Cho Y, Hawley CM, et al. Early and late patient outcomes in urgent-start peritoneal dialysis. Perit Dial Int. 2017;37:414-419.

74. Lomonte C, Basile C. Preoperative assessment and planning of haemodialysis vascular access. Clin Kidney J. 2015;8:278-281.

75. Letachowicz K, Szyber P, Golebiowski T, et al. Vascular access should be tailored to the patient. Semin Vasc Surg. 2016;29:146-152.

76. Vanholder R, Glorieux G, Eloot $\mathrm{S}$. Once upon a time in dialysis: the last days of Kt/V? Kidney Int. 2015;88:460-465.

77. Schroeder EB, Yang X, Thorp ML, et al. Predicting 5-year risk of RRT in stage 3 or 4 CKD: development and external validation. Clin J Am Soc Nephrol. 2017;12:87-94. 